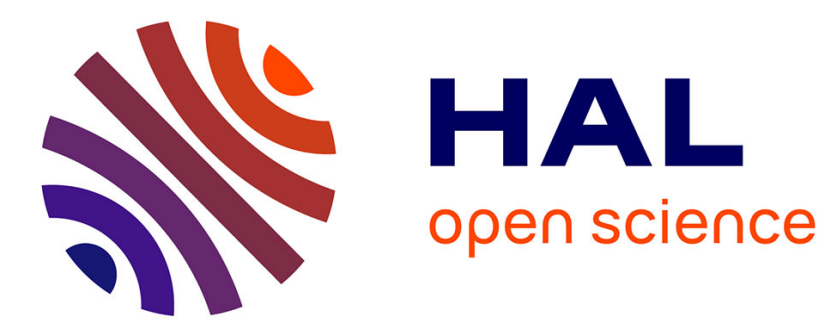

\title{
A mechanism for the sphere/tube shape transition of nanoparticles with an imogolite local structure (imogolite and allophane)
}

Antoine Thill, Pierre Picot, Luc Belloni

\section{- To cite this version:}

Antoine Thill, Pierre Picot, Luc Belloni. A mechanism for the sphere/tube shape transition of nanoparticles with an imogolite local structure (imogolite and allophane). Applied Clay Science, 2017, 141, pp.308-315. 10.1016/j.clay.2017.03.011 . cea-01487701

HAL Id: cea-01487701 https://hal-cea.archives-ouvertes.fr/cea-01487701

Submitted on 13 Mar 2017

HAL is a multi-disciplinary open access archive for the deposit and dissemination of scientific research documents, whether they are published or not. The documents may come from teaching and research institutions in France or abroad, or from public or private research centers.
L'archive ouverte pluridisciplinaire HAL, est destinée au dépôt et à la diffusion de documents scientifiques de niveau recherche, publiés ou non, émanant des établissements d'enseignement et de recherche français ou étrangers, des laboratoires publics ou privés. 


\title{
A mechanism for the sphere/tube shape transition of nanoparticles with an imogolite local structure (imogolite and allophane).
}

\author{
A. Thill*, P. Picot and L. Belloni \\ LIONS, NIMBE, CEA, CNRS, Université Paris-Saclay, 91191 Gif sur Yvette, France. \\ *corresponding author : antoine.thill@cea.fr
}

\begin{abstract}
Imogolite and allophane are two strongly curved nanominerals. Indeed, imogolite has a tubular shape with a diameter of only $2.5 \mathrm{~nm}$. Allophane is often presented as a spherical nanostructure with a diameter of about $5 \mathrm{~nm}$. The mechanism explaining the strong curvature of these two nanominerals has been extensively discussed. However, whether or not these two nanomaterials are related to each other is not clear and the mechanism responsible for the selection between the two different shapes is not well established. In this article, we propose that imogolite and allophane are nanopolymorphs of the imogolite local structure and that the transition from spherical to tubular shape occurs at an early stage of the precipitation because of edge stress in proto-imogolite. This hypothesis for the shape selection is supported by the use of a nanomechanical model tuned to mimic the main characteristics of imogolitelike nanomaterials.
\end{abstract}

Keywords : imogolite; allophane; crystal polymorph; molecular dynamic

\section{Introduction}

It is commonly observed that a material can adopt a variety of structures. For simple elements, this is the notion of allotropy. For minerals, this is the notion of polymorphism. Carbon is an emblematic example for allotropy as it can form both diamond and graphene. At the nanoscale, carbon atoms with a sp2 hybridization can arrange in the form of nanospheres (fullerene), nanotubes of various chirality and wall number, sheets and other exotic shapes recently called nanoallotropes (Georgakilas et al., 2015). The perfect control of the different shapes is however difficult for carbon nanoallotropes. The same phenomena is observed for aluminosilicates presenting an imogolite-like local structure (ILS) with composition $\mathrm{Al}_{2} \mathrm{SiO}_{3}(\mathrm{OH})_{4}$. In this case, it is possible to talk about nanopolymorphs as imogolite and allophane can enter the category of nanocrystals. Allophane having an ILS are described as 4-5 $\mathrm{nm}$ 
spheres(Henmi and Wada, 1976) while imogolites are very long nanotubes with a monodisperse diameter between 2 and $2.8 \mathrm{~nm}$ depending on the formation conditions. Imogolite are believed to be obtained from proto-imogolites, which are described as curved pieces of ILS with an average edge size of $5 \mathrm{~nm}$ (Levard et al., 2010). Unlike carbon, very efficient control over the different shapes is possible through adapted synthesis.

The ILS is a very original structure for clay minerals. Generally, the silicon atoms form a polymerized tetrahedral sheet in which each $\mathrm{Si}$ atom is linked to a dioctahedral (e.g. Kaolinite or Halloysite) or trioctahedral (e.g. Chrysotile) sheet by a single Si-O-Al or Si-O-Mg bond. This is not the case in the ILS where Si atoms are isolated and form three bonds within the lacuna of the dioctahedral aluminum sheet (Cradwick et al., 1972). The ILS is characterized by a strong curvature. Imogolite has a well-defined radius going from $2 \mathrm{~nm}$ for natural imogolite to $2.8 \mathrm{~nm}$ for synthetic nanotubes. It is possible to vary the size of imogolite by changing the temperature (Wada, 1987), the type of chemical precursors(Yucelen et al., 2012) or by replacing Si atoms by Ge atoms(Wada and Wada, 1982). However, the nanotubes formed in the same conditions have all the same diameter. The reasons explaining this well-defined curvature have been discussed soon after the imogolite discovery (Farmer et al., 1977). The early explanation was that the tetrahedric Si site has to create bonds in the lacuna of the dioctahedral sheet which is larger than its relaxed size. Indeed, if one consider gibbsite as a reference structure for uncurved 2D dioctahedral Al sheet, the $\mathrm{O}-\mathrm{O}$ distance ( edge length of the Al octahedron) is $2.9 \AA$ whereas the O-O edge distance in the relaxed Si tetrahedron is $\sim 2.6 \AA$. This size mismatch is the most obvious reason for the spontaneous curvature associated with the ILS. However, the size mismatch between the relaxed Si tetrahedron and the $\mathrm{O}-\mathrm{O}$ distance of the dioctahedral $\mathrm{Al}$ sheet is not sufficient to explain the very strong spontaneous curvature of imogolite. It is also important to consider the role of the electrostatic charges and hydrogen bonds ( $\mathrm{H}$ Bond). The role of the internal $\mathrm{Si}-\mathrm{OH}$ groups in the stability of imogolite nanotubes has been explored by density functional theory (DFT) (Lee et al., 2011). It is demonstrated that the $\mathrm{H}$ bond network inside the nanotubes contribute to the stability in size and to the selection of a preferential ZigZag organization(Demichelis et al., 2010; Lee et al., 2011).

An indirect experimental confirmation of the role of an internal $\mathrm{H}$ bond network has been obtained by Bottero et al. (2011). Indeed, they obtained a synthetic nanomineral having an ILS by using triethoxymethylsilane as a silicon source in the co-precipitation step. The obtained nanoparticles are still curved; the concave part being covered by $\mathrm{Si}_{-} \mathrm{CH}_{3}$ groups instead of $\mathrm{Si}-\mathrm{OH}$ making strongly hydrophobic nanoconfined cavities. This chemical modification prevents the formation of an internal network of $\mathrm{H}$ bond. It is observed that these nanotubes are significantly larger than their natural $\mathrm{Si}-\mathrm{OH}$ analogue (Amara et al., 2015) confirming indirectly the important role of $\mathrm{H}$ bond for the determination of their spontaneous curvature.

The spontaneous curvature of imogolite has been studied by many different types of molecular dynamic (MD) and DFT techniques. It is always concluded that it exists a spontaneous curvature of minimum energy (Tamura and Kawamura, 2002; Alvarez-Ramirez, 2007; Li et al., 2008; Demichelis et al., 2010) even if the model may predict different values. An elegant analytical expression has been proposed considering a dissymmetric surface tension on a flexible thin material (Guimarães et al., 2007). This expression seems to capture the essential physics behind the curvature of ILS and nicely describes the 
shape of the energy minimum obtained by different simulations. Based on such simplified analytical expressions, it is possible to successfully explain the single to double wall transition in germanium based imogolite (Thill et al., 2012). A generalized version has been used to describe the structure and thermodynamic of curved clay minerals (Belloni and Thill, 2016). While this phenomenological model enables to nicely relate the spontaneous curvature to the mechanical properties of ILS, it does not allow to predict the sphere/tube transition essentially because it neglects border effects.

DFT simulations have been coupled to experiments to propose a mechanism for the selection of the two types of curvature (spherical and tubular). It is concluded that the ions and $\mathrm{pH}$ seems to play an important role (Abidin et al., 2007; Bac et al., 2009). The use of $\mathrm{Si}-\mathrm{CH}_{3}$ groups for the internal surface of the ILS nanominerals maintains a spontaneous curvature without the formation of $\mathrm{H}$ bond network and electrostatic interactions. Modifications of the synthesis proposed by Bottero et al. (2011) allow to obtain both shapes with hybrid $\mathrm{Si}_{-} \mathrm{CH}_{3}$ ILS. Obviously, in this case, the transition from spherical to tubular morphology cannot be linked to the deprotonation of $\mathrm{Si}-\mathrm{OH}$ groups or the formation of internal $\mathrm{H}$ bond network and this result calls for an alternative explanation.

In this work, we propose a hypothesis for the shape transition saying that it is linked to a competition between surface and border tensions in proto-imogolite. To test this hypothesis, we use a simplified computer model to explore the curvature of proto-imogolites of various sizes. We used a hybrid model which is close to the atomistic description of the ILS and thus allows us to account for border effects. We will not try to perform a realistic mechanical description of imogolite or allophane as in MD or DFT models. We rather explore a general problem using a simplified mechanical system. The questions of interest are: i) Is it possible to correctly mimic the mechanical behavior of imogolite with such a simplified description? ii) Is it possible to observe a transition from a spherical to a tubular shape without interaction with the solvent? We will then discuss the implications of this simplified curvature mechanism on the formation mechanism of the different ILS nanominerals.

\section{Methods}

\subsection{Numerical model of the hybrid ILS}

In the Guimarães et al. (2007) model, a plastic 2D thin material is subjected to different surface tensions on its two sides. In this case, the mechanical energy per unit surface as a function of the radius of curvature $\mathrm{R}$ of the $2 \mathrm{D}$ material reads

$E=\frac{Y h^{3}}{3 R^{2}}-\frac{\sigma h}{R}+\sum$

where $\mathrm{Y}$ is the Young modulus of a 2D slab of thickness $2 \mathrm{~h}, \sigma$ is the difference in surface tension between the two surfaces and $\Sigma$ is a constant. This equation can be identified with a quadratic expression of the form 
$E=E_{0}+\frac{K_{c}}{2}\left(\frac{1}{R}-\frac{1}{R_{0}}\right)^{2}$

with $\mathrm{K}_{\mathrm{c}}=2 \mathrm{Yh}^{3} / 3$ and $\mathrm{R}_{0}=2 \mathrm{Yh}^{2} / 3 \sigma$. Considering the estimation of the Young modulus of the ILS which is predicted to be of the order of $300 \mathrm{GPa}$ and considering the thickness of the dioctahedral sheet to be $2 h=2.9 \AA, K_{c}$ is about $150 \mathrm{kT}$. This simple expression enables to reproduce the shape of the curve $E=f(R)$ obtained by advanced DFT simulations. For example, the results of Guimarães et al. (2007) for the energy of imogolite of various radius can be fitted with eq. 2 using $K_{c}=156 \mathrm{kT}$ and $\mathrm{R}_{0}=9.2 \AA$.

Here, a simplified description of the ILS is used consisting in an assembly of flexible octahedra and tetrahedra (Figure 1). Harmonic bond and angle potentials are used to tune the shape and flexibility of the structure(Konduri et al., 2006). The assembly of flexible octahedra will play the role of the flexible 2D material and the difference in surface tension will be induced by the bonding on only one side of flexible tetrahedra. The amplitude of the surface tension difference is controlled by the size and rigidity of the tetrahedra.

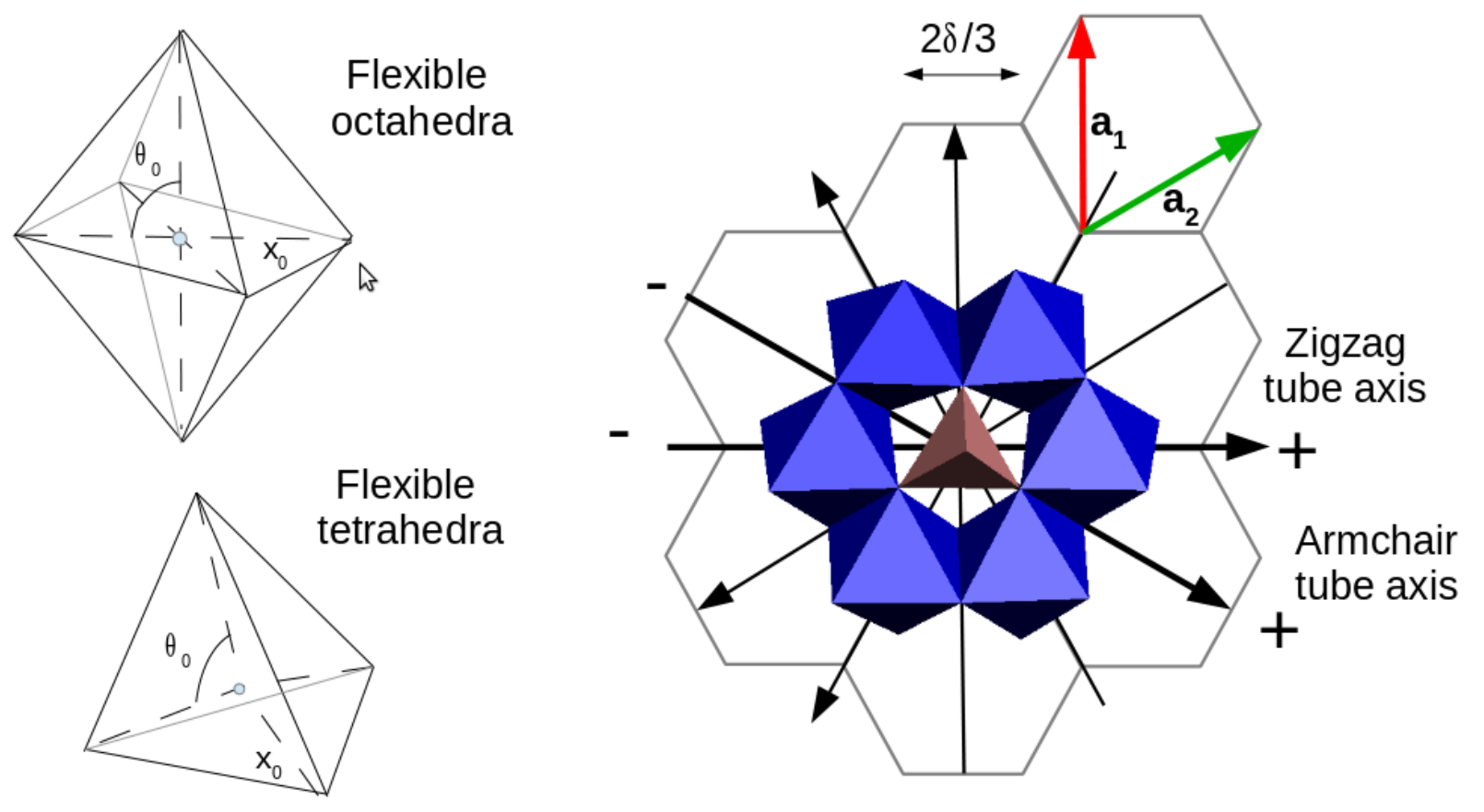

Figure 1. The model is based on flexible octahedra and tetrahedra having potential energy for centervertice stretching $E_{s}=1 / 2 K_{s}\left(x-x_{0}\right)^{2}$ and vertice-center-vertice bending $E_{b}=1 / 2 K_{b}\left(\theta-\theta_{0}\right)^{2}$. The values $K_{s}, x_{0}, K_{b}$, and $\theta_{0}$ are not the same for octahedra and tetrahedra (see text). The octahedra are linked by edges in a Gibbsite-like 2D hexagonal structure. The tetrahedra are linked to 6 neighbors octahedra through three bonds. The positions in the 2D Gibbsite like structure are conveniently obtained with the two unit vectors $\mathbf{a}_{\mathbf{1}}$ and $\mathbf{a}_{\mathbf{2}}$. 
The octahedra are sharing edge links as in a gibbsite-like structure. In the lacuna of the 2D gibbsite-like structure the tetrahedra share three common vertices with the octahedra. The centers of the octahedra and tetrahedra mimic the $\mathrm{Al}$ and $\mathrm{Si}$ atoms in the ILS, respectively. The vertices of octahedra and tetrahedra correspond to the positions of $\mathrm{O}$ atoms in the ILS. The interactions in the structure are computed using exclusively harmonic potentials for the stretching and bending of the center-vertices bonds and angles, respectively. The energy for the stretching term is given by $E_{s} \sim 1 / 2 K_{s}\left(x-x_{0}\right)^{2}$ where $K_{s}$ is the stretching rigidity of the bond and $x_{0}$ its equilibrium distance. For the bending energy $E_{b} \sim 1 / 2 K_{b}\left(\theta-\theta_{0}\right)^{2}$ where $K_{b}$ is the bending rigidity and $\theta_{0}$ the equilibrium angle. We have considered a single object of a given size without periodic boundary conditions. Indeed, usual simulations with periodic boundary conditions impose a tubular shape. The structure is composed only of octahedra and tetrahedra and only tetrahedra bonded to three octahedra are considered. To prevent interpenetration of distant polyhedra upon curvature of very large structures, non-bond interactions are computed using Lennard-Jones potentials for points separated by at least three bonds. The Lennard Jones parameters are taken from the CLAYFF force field(Cygan et al., 2004) considering the vertices as $O$ atoms and the centers of tetrahedral and octahedra as Si and Al atoms, respectively.

Konduri et al. (2006) have studied the curvature of $\mathrm{Al} / \mathrm{Si}$ and $\mathrm{Al} / \mathrm{Ge}$ imogolite using such simplified description with harmonic potentials. They show that this type of model can mimic the shape of the strain energy as a function of the radius of imogolite. From the comparison with MD simulations, they proposed values for the stretching rigidity and bond equilibrium distance of $\mathrm{K}_{\mathrm{s}}=6.06 \mathrm{eV} . \AA^{-2}$ and $\mathrm{x}_{0}=1.59$ $\AA$ for Si-O, $\mathrm{K}_{\mathrm{s}}=2.52 \mathrm{eV} . \AA^{-2}$ and $\mathrm{x}_{0}=1.97 \AA$ for Al-O. In their analysis, the bending angles of O-Si-O and OAl-O were considered fixed at respectively $109.5^{\circ}$ and $90^{\circ}$. These approximations certainly induce an underestimation of the stretching rigidities. For example, the harmonic approximation of the CLAFF potential around the energy minimum gives a stretching rigidity of $\mathrm{K}_{\mathrm{s}}=40.5 \mathrm{eV} . \AA^{-2}$ and a distance at minimum energy of $1.43 \AA$. Such rigidity is in better agreement with the IR wavenumber around 1000 $\mathrm{cm}^{-1}$ for the absorbance attributed to the stretching of the Si-O bond. In the CLAYFF, the bending rigidity of the O-Si-O and O-Al-O angles is 1.302 eV.rad ${ }^{-2}$. Thus, compared to the Konduri et al. (2006) approximation, MD simulations based on the CLAYFF tend to model the structure with relatively rigid distances and rather flexible angles. In the following, we will adopt the bending rigidity proposed by the CLAYFF and consider the stretching rigidity and equilibrium distance as adjustable parameters of our model. To choose appropriate values, we will rely on well-known characteristics of the imogolite nanotubes i.e. the distance between successive tetrahedra in a Zig-Zag nanotube structure and the correct shape of the $E=f(R)$ curve.

Two different initial configurations are considered (González et al., 2014). First, a tubular initial configuration (TIC) will be used to choose the model parameters. Then, a hypothetical planar initial configuration (PIC) where the octahedra form a flat sheet and the tetrahedra are initially stretched. This simplified description is not appropriate for an accurate description of the ILS as it neglects the interaction with the solvent, the long range electrostatic interactions and the formation of $\mathrm{H}$ bond networks. But if it can reproduce the correct shape of the $E=f(R)$ curve, we believe it can illustrate our hypothesis regarding the mechanical control of the sphere/tube shape transition. 


\subsection{Tubular initial configuration}

Like in many theoretical works concerning imogolite, the formalism to describe the structure of carbon nanotubes is used. The nanotubes are noted $(n, m)$ where $n$ and $m$ are the coordinates of the rotation vector $\mathbf{R}=n \mathbf{a}_{1}+m \mathbf{a}_{2}$ in an hexagonal network with $\left|\mathbf{a}_{1}\right|=\left|\mathbf{a}_{2}\right|=2 \delta / 3^{1 / 2}$ (see figure 2 ) where $\delta$ is half the repeat distance along the c-axis of the Zig-Zag nanotube. The norm of $\mathbf{R}$ gives the nanotube perimeter and thus the nanotube radius is $r=|R| / 2 \pi$. As the ILS has a thickness of about $6 \AA$, the actual radius depends on a reference position in the layer. In the following, the radius is calculated considering the plane containing the centers of the octahedra.

It is interesting to note that the imogolite structure is oriented (see figure 1 and 2). Indeed, because of the presence of the tetrahedral Si atom, the structure is not symmetric upon a rotation of $180^{\circ}$. Therefore, each imogolite nanotube has two distinct sides noted + and -. $(n, m)+$ and $(n, m)$ - tubes can be built depending on the alignment of the structure with respect to the $z$ axis. Implications of this orientation have never been discussed in the literature. For example, it has been demonstrated that a significant part of the nanotubes growth occurs through tip-tip collisions (Maillet et al., 2011; Yucelen et al., 2013). In these tip-tip collisions, it is of course needed that the two colliding tubes have the same rotation vector but they also need to be aligned in the same direction. The tip on the + side of the nanotube has to come in contact with the tip on the - side for the creation of bonds to be possible. This fact is another contribution explaining the slow growth of the nanotubes even at high concentrations.

\subsection{Planar initial configuration}

In PIC, the octahedra are placed on a 2D hexagonal lattice of unit vector $\mathbf{a}_{\mathbf{1}}$ and $\mathbf{a}_{\mathbf{2}}$. The borders of the 2D PIC are defined by the vector $\mathbf{R}=\mathbf{n a}_{1}+\mathrm{ma}_{2}$ of norm $L_{R}$ and the perpendicular vector $T$ of norm $L_{T}$. When the structure is defined in such a way, the coordination of the border atoms may not be satisfied. As we have chosen to work with only octahedra and tetrahedra bonded to three octahedra, the coordination of atoms is completed and whenever a tetrahedron is not bonded to three octahedra, it is removed. The 2D PICs are described by the $(n, m)$ indices defining $R$ and the ratio between $L_{R}$ and $L_{T}$. The behavior of PICs of various sizes and two orientations of the $\mathbf{R}$ vector $(n=i, m=0)$ and $(n=m=i)$ with i between 2 and 20 are considered. The values $L_{R}$ and $L_{T}$ cannot be equals as for $(n=i, m=0) L_{R} \sim \delta / 3^{1 / 2}$ and $L_{T} \sim \delta$ and for $(n=$ $m=i) L_{R} \sim \delta$ and $L_{T} \sim \delta / 3^{1 / 2}$. We choose $i$ and $L_{T}$ to have $L_{R} / L_{T}$ as close as possible to 1 in order to avoid any preferential axis initially. The figure 2 shows examples of $(14,0)$ and $(8,8)$ PIC structures and the corresponding tubes obtained after rolling the PIC. 

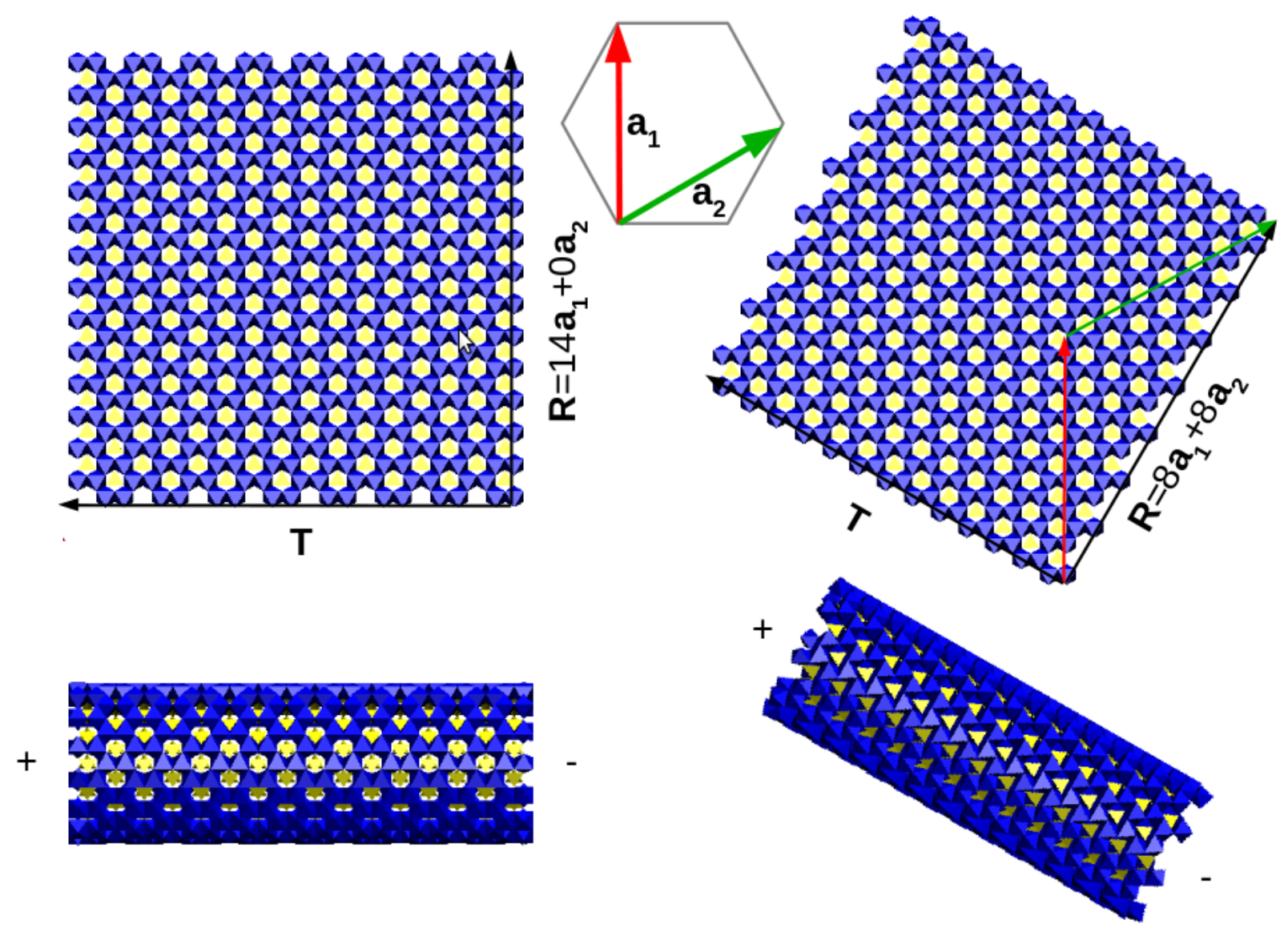

Figure2. The construction of PIC and TIC uses the convention adopted for the description of carbon nanotube structure. The hexagonal lattice of unit vector $\mathbf{a}_{\mathbf{1}}$ and $\mathbf{a}_{\mathbf{2}}$ is used to define two vectors $\mathbf{R}=$ $n a_{1}+m a_{2}$ of norm $L_{R}$ and a perpendicular vector $\mathbf{T}$ of norm $L_{T}$. Examples of a $(14,0)$ and $(8,0) L_{R} / L_{T} \sim 1$ PIC are shown. The tubes obtained with the rolling of the two PIC structures are also shown. For PIC, $L_{R}$ and $L_{T}$ are the length of the borders, in TIC $L_{R}$ is the perimeter of the tube and $L_{T}$ its length. The nanotubes are oriented due to the presence of the tetrahedron (see text).

\subsection{Numerical simulations}

The initial configurations are used with the LAMMPS code to compute the zero Kelvin minimum energy using a conjugated gradient minimization (Plimpton, 1995). MD trajectories are also computed using a thermostat at $300 \mathrm{~K}$ to follow the shapes of various TIC and PIC configurations for $120 \mathrm{ps}$. For the TIC, the average energy is computed for zigzag configurations from $(8,0)$ to $(25,0)$ and for armchair from $(6,6)$ to $(14,14)$. The radius of the nanotube is computed at the plane containing the centers of the octahedra. $\delta$ is obtained from the length of the nanotube and the number of repeated circumferences.

For the PIC, the two principal radii of curvature are computed at the surface containing the $\mathrm{N}$ centers of the octahedra located at absolute positions $x_{i}, y_{i}, z_{i}$, with $i=1, N$. Nothing is known a priori about the location, orientation or shape of this surface. The first step consists in shifting the atomic positions 
relative to that of the center of mass $G$, so to replace $x_{i}$ by $x_{i}-x_{G}$, etc. The matrix of inertia is constructed according to:

$$
\left(\begin{array}{ccc}
Y^{2}+Z^{2} & -X Y & -X Z \\
-X Y & X^{2}+Z^{2} & -Y Z \\
-X Z & -Y Z & X^{2}+Y^{2}
\end{array}\right)
$$

where one defines $X^{2}=\sum_{i} m_{i} x_{i}^{2}, X Y=\sum_{i} m_{i} x_{i} y_{i}, \ldots$ This matrix is diagonalized into $\left(\begin{array}{ccc}I_{1} & 0 & 0 \\ 0 & I_{2} & 0 \\ 0 & 0 & I_{3}\end{array}\right)$ in order to derive the three principal moments $I_{1}, I_{2}, I_{3}$ and the three corresponding principal axis $\boldsymbol{u}_{1}, \boldsymbol{u}_{2}, \boldsymbol{u}_{3}$. The idea of seeing the whole collection of atoms as a weakly curved surface is meaningful only if the excursion along one of the main axis, say $\boldsymbol{u}_{3}$, remains small compared to the two others, so only if one of the main moments is detected in practice to be close to the sum of the two others, say $I_{3} \approx I_{1}+I_{2}$. If this happens, the surface deviates only weakly from a plane of normal $\boldsymbol{u}_{3}$ and the notion of local curvatures is relevant. The absolute atomic coordinates are then transformed into the coordinates $v_{1, i}, v_{2, i}, v_{3, i}$ in the local frame characterized by the vectors $\boldsymbol{u}$. Finally, these positions are best fitted with an arc of paraboloid, $v_{3}=a v_{1}{ }^{2}+b v_{2}{ }^{2}+c$, and the required principal radii of curvature are simply $R_{1}=1 / 2 a$ and $R_{2}=1 / 2 b$.

\section{Results and Discussions}

The shape and elasticity of the structures in the proposed model depend on the choice of the bending and stretching potentials of octahedra and tetrahedra. To choose values able to reproduce the main behavior of hybrid ILS, we have compared the structure predicted by the model with experimentally accessible imogolite characteristics i.e. the spontaneous curvature and rigidity $(E=f(R))$ and the repeat distance $2 \delta$ along the c-axis of the tube. Then the model is applied to PIC of various size to mimic the curvature of proto-imogolite.

\subsection{Parameters to reproduce the curvature, rigidity and c-axis periodicity of the tubes.}

The relationship between the number of $\mathrm{Al}$ atoms in the imogolite nanotube section and the energy of the nanotube has been studied by several authors ( Tamura and Kawamura, 2002; Alvarez-Ramirez, 2007; Creton et al., 2008; Li et al., 2008; Zhao et al., 2009; Demichelis et al., 2010). The curves may have different values but the trends and shape are always very similar with the existence of a spontaneous curvature and a shape nicely described by the analytical model proposed by Guimarães et al. (2007). Our model will not be able to reproduce the radius of imogolite with realistic bond parameters as the curvature is partly linked to internal $\mathrm{H}$ bond network and electrostatic interactions. However, we should be able to obtain an energy curve giving a radius of minimum energy close to the hybrid $\mathrm{Si}-\mathrm{CH}_{3}$ imogolite where all these effects are not active. According to Bottero et al. (2011) the external radius of hybrid imogolite determined by XRD is $15.1 \AA$. Amara et al. (2015) obtained exactly the same radius with Small 
Angle X-ray Scattering (SAXS). This corresponds to a radius of $13 \AA$ for the plane containing the Al atoms. Recently, DFT model were used to obtain the curve $E=f(R)$. They obtain a surprisingly higher rigidity 3 times higher than $\mathrm{Si}-\mathrm{OH}$ imogolite and a radius of $12.3 \AA$ at the Al plane. Figure 3 is the energy curves for Zig-Zag tubes from $(10,0)$ to $(20,0)$ and Armchair tubes from $(6,6)$ to $(14,14)$ as a function of the radius at the center of the octahedra (Al atoms) taking $\mathrm{x}_{0}=1.4 \AA, 2.15 \AA$ and $\mathrm{K}_{\mathrm{s}}=81 \mathrm{eV} . \AA^{-2}, 55 \mathrm{eV} . \AA^{-2}$ for the equilibrium distances and stretching rigidities of the center-vertice of respectively tetrahedra and octahedra. The curves are fitted with equation 2 to obtain the radius at minimum energy $R_{0}$ and the rigidity $K_{c}$. The same $R_{0}$ for Zig-Zag and Armchair nanotubes is obtained at $13 \AA$ in very good agreement with the experimental data. The rigidity $\mathrm{K}_{\mathrm{c}}$ is almost the same for the two configurations (125 and $120 \mathrm{kT}$ for respectively Zig-Zag and Armchair). This rigidity is slightly less than the one predicted for imogolite Si$\mathrm{OH}(\sim 150 \mathrm{kT})$.

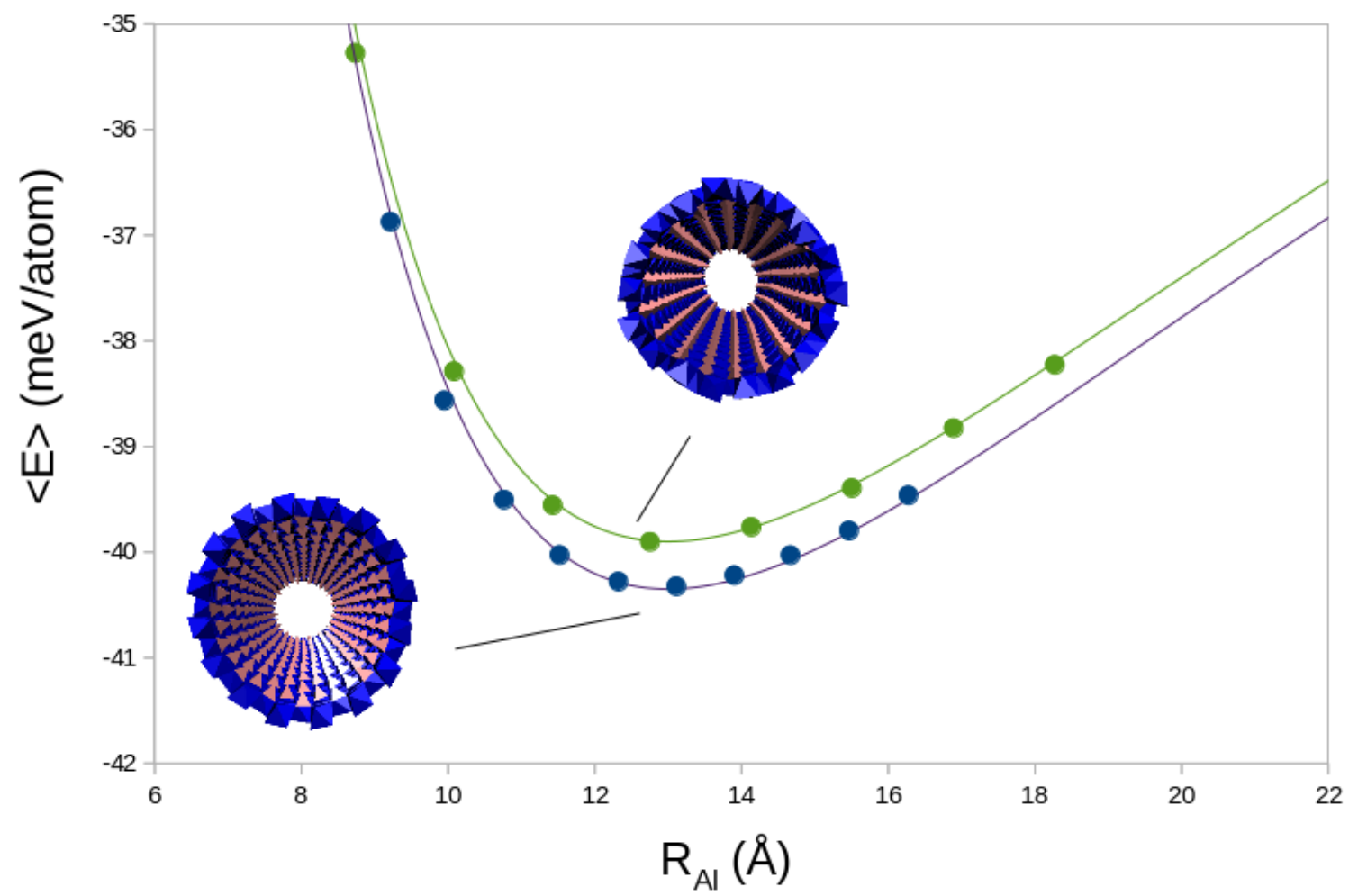

Figure3. Average potential energy of Zig-Zag and Armchair nanotubes with radius between 8 and $20 \AA$. The energy is given in meV/atom where the atom number corresponds to vertices (counted only once when shared) and centers of tetrahedral and octahedral. The lines are fits of the curves $\langle E\rangle=f(R)$ with the equation $\langle E\rangle=E_{0}+0.5 K_{c}\left(1 / R-1 / R_{0}\right)^{2}$.

The case of the nanotube periodicity in the c-axis $\delta$ is less often discussed in detail. Indeed, as most of the simulations are using periodic boundary conditions, $\delta$ is generally a fixed parameter. The experimental value of $\delta$ can be obtained by SAXS experiment. Indeed, a small reflection is obtained for a $2 \theta$ Bragg angle of $20.3^{\circ}$ ( $\mathrm{Cu} \mathrm{K} \alpha$ ) corresponding to a second order reflection for the repeat distance of $2 \delta=8.72 \AA$ (Russell, 1969; Taché et al., 2016). With the present simplified model, the simulation is 
performed on a single nanotube of finite size. The modifications of the bending and stretching rigidities have thus an impact on both the curvature and $\delta$. We have examined the finite size effect on the nanotube strain energy. Indeed, the borders offer an additional relaxation possibility through an additional local curvature. Figure 4 presents the average strain energy and the mean $\delta$ as a function of the nanotube length from 10 to $200 \AA$.

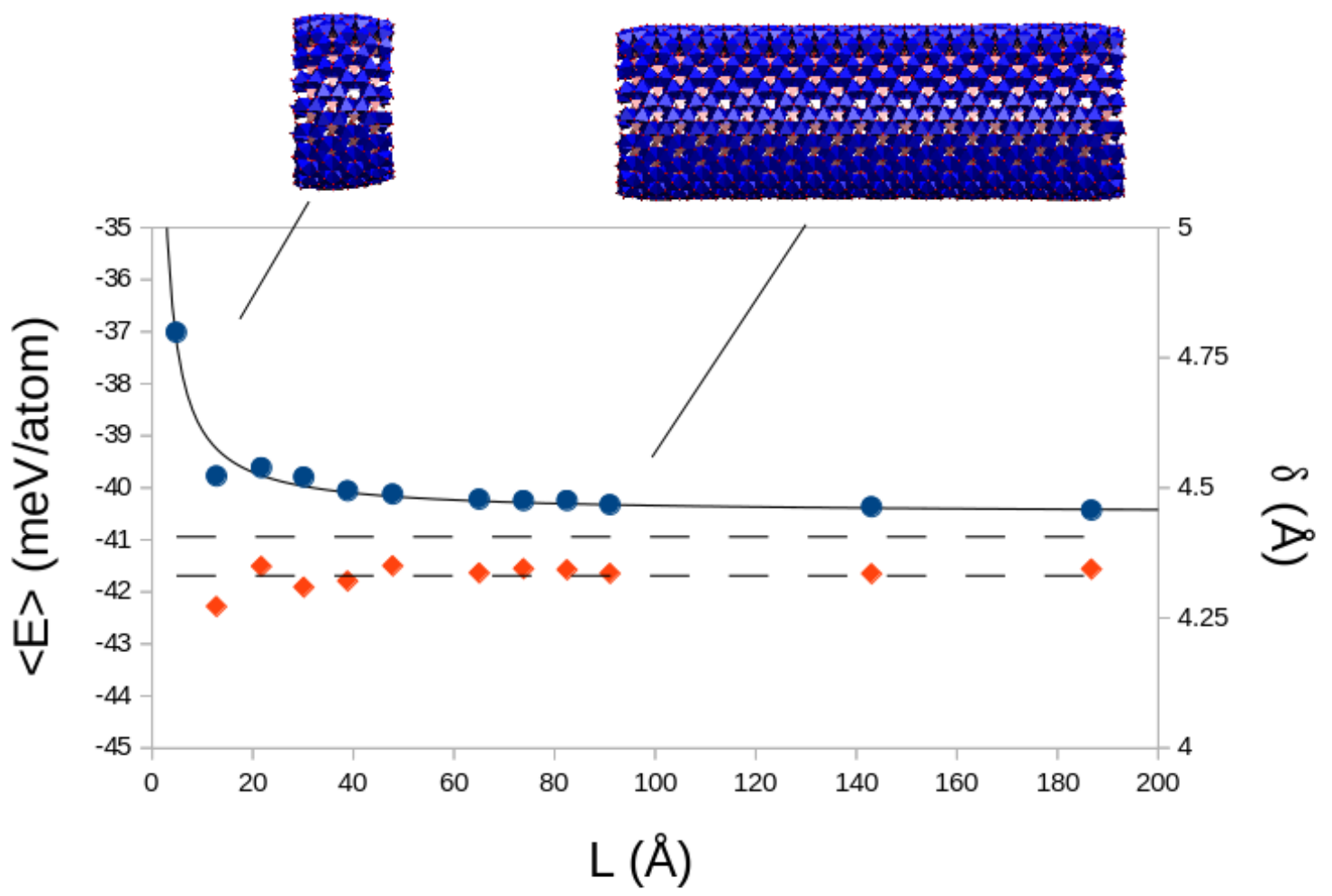

Figure 4. Average potential energy $<\mathrm{E}>$ in $\mathrm{meV} /$ atom (atom number obtained as described in figure 3 ) and repeat distance $\delta$ in the c-axis for nanotubes of increasing length between 10 and $200 \AA$. The dotted line corresponds to the experimentally observed $\delta$ value for hybrid imogolite. The full line is given by equation $\langle\mathrm{E}\rangle=\left\langle\mathrm{E}_{\infty}\right\rangle+\mathrm{K}_{\mathrm{b}} / \mathrm{L}$.

The average energy follows a trend $\langle E\rangle_{L}=\langle E\rangle_{\infty}+K_{b} / L$ where $K_{b}$ corresponds to an excess border energy. The effect of the finite size is noticeable on the shape of the nanotube border with a slight decrease of the radius. This effect is not negligible for almost the whole range of size of practical interest. Indeed, in classical imogolite synthesis, the average length of the nanotube reaches about $200 \mathrm{~nm}$. The same effect is observed for the $\delta$ value. A slight decrease of $\delta$ is associated to the shortest nanotubes.

The simple description of the ILS with flexible octahedra and tetrahedra is able to reproduce the mechanical behavior of the nanotube with the correct shape of the energy as a function of the radius. Even if the goal is not to approach an accurate atomistic description of the ILS, it is interesting to note 
that the parameters able to mimic the strain behavior give the correct value for the repeat distance $\delta$ along the c-axis of the nanotube.

\subsection{Curvature of the PIC as a function of their size.}

Using this set of parameters, we have performed MD simulations on PIC of growing size. The figure 5 shows the average potential energy as a function of $\left(L_{R}+L_{T}\right) / 2$. On the same graph, the average values of the two principal curvatures are also plotted.

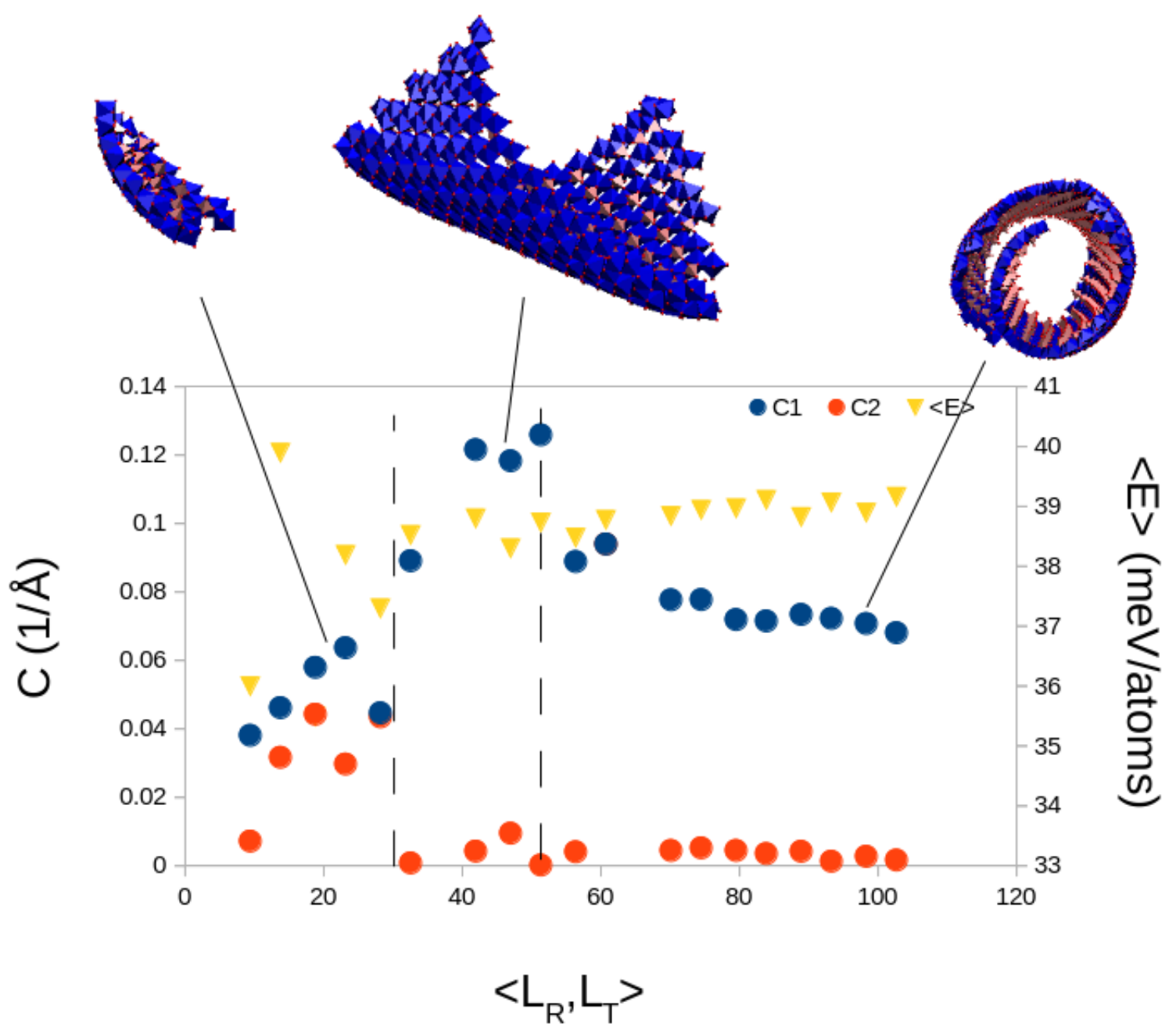

Figure 5. Average curvatures $\left\langle\mathrm{C}>\right.$ and average stretching energy $\left\langle\mathrm{E}_{\mathrm{b}}\right\rangle$ for $\mathrm{PIC}$ of increasing size from 5 to $100 \AA$. The decrease of the largest curvature for the sizes above the threshold may be partly due to a failure of the algorithm for the largest PIC giving spirals after rolling.

The energy of the PIC increases with the size. The rate of increase is however not constant. For the smallest PIC up to $\left(L_{R}+L_{T}\right) / 2=40 \AA$, the energy increases at a fast rate with the size. Above this value, the rate significantly decreases. For the principal curvatures, a modification also occurs at the same size. Before $\left(L_{R}+L_{T}\right) / 2=40 \AA$, the two principal curvatures $C_{1}$ and $C_{2}$ follow similar trend with values increasing 
from 0.01 to $0.06 \AA^{-1}$. After the size threshold, the two principal curvatures evolve in opposite direction giving a maximum curvature with $C_{1} \sim 0.1 \AA^{-1}$ similar to the spontaneous TIC curvature and a minimum curvature $C_{2}$ which vanishes. Snapshots of the PIC during the MD simulation clearly show two distinct morphologies before and after the size threshold. Before the threshold, the PIC behaves like a portion of sphere without clear privileged direction. After the threshold, an axis of low curvature can be identified. The shape transition is even clearer if one plots the two principal curvatures during the MD simulation for the two situations (below and above the size threshold). Figure 6 presents the curvature evolution as a function of time for $\left(L_{R}+L_{T}\right) / 2=20 \AA$ and $60 \AA$.
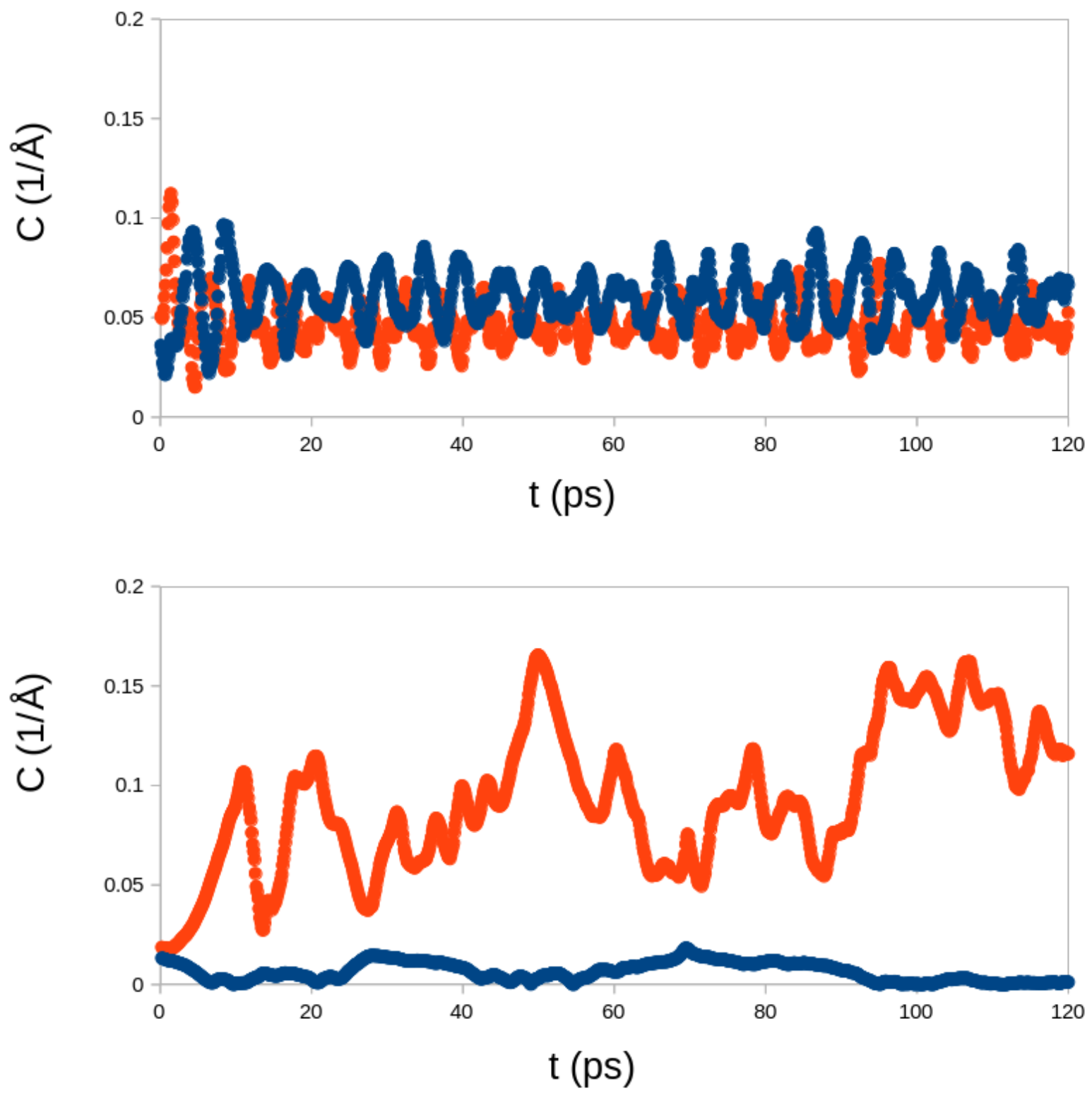

Figure 6. Time evolution of the two principal curvatures for PIC of $20 \AA$ (top graph) and $60 \AA$ (bottom graph) which are respectively bellow and above the sphere/tube size threshold. 
In both case, fluctuations of the principal curvatures with time are observed. For the smallest PIC, $\mathrm{C}_{1}$ and $C_{2}$ fluctuate in opposite phase with the same amplitude between 0.025 and $0.05 \AA^{-1}$. The largest PIC shows a clear separation between the two principal curvatures values. One value fluctuates at about $0.1 \AA^{-1}$ whereas the other one is vanishing. So our model of the PIC curvature clearly demonstrates that a transition from a spherical to a tubular shape occurs as the size increases. For the particular case of the selected parameters of the model, the threshold is occurring for a size of $4 \mathrm{~nm}$ which is slightly smaller than the average proto-imogolite size of $5 \mathrm{~nm}$ obtained from SAXS and DLS measurements (Levard et al., 2010; Mukherjee et al., 2005).

\subsection{Implications for imogolite, allophane synthesis}

Based on this result, we propose an alternative hypothesis which could explain the formation of allophane or imogolite. The transition could be due to modifications of the nucleation stage of the protoimogolite. Indeed, the precipitation of an amorphous phase of type $\mathrm{HAS}_{\mathrm{A}}$ (Exley et al., 2002) and its further internal restructuration could well produce proto-imogolites of various average sizes depending on the synthesis conditions. Concentration, $\mathrm{pH}$ and type of Si precursors seem to potentially control the formation of allophane or imogolite (Denaix et al., 1999). These parameters may influence the average size of proto-imogolites. Three scenarios can be envisioned. First, the average size of the proto-imogolite is well below the threshold for the sphere/tube shape transition. In this case, spherical proto-imogolite will probably self-assemble and eventually lead to the formation of allophane. In the second situation, the obtained proto-imogolites have an average size well above the threshold for the sphere/tube shape transition. In this case, only tubular shaped proto-imogolites interact and self-assemble into imogolite. An intermediate situation may exist when the average size of the proto-imogolite is roughly equal to the threshold for the sphere/tube shape transition. In this case, both spherical and tubular proto-imogolite will co-exist (Henmi and Wada, 1976). This particular case could explain why in some synthesis, imogolites tend to co-exist with proto-imogolites even after a prolonged growth period (Koenderink et al., 1999). While it is clear that tubular shaped proto-imogolites could not easily assemble into a spherical shape, it is less obvious to understand why proto-imogolites below the threshold will not selfassemble in a way promoting their shape transition once they get larger than the threshold for the sphere/tube shape transition. For our scenario to be possible, we have to consider i) a separation of a "nucleation" and growth stage of proto-imogolite and ii) an incompatibility between proto-imogolites below and above the shape transition during the growth stage. Validation of this scenario will require the exploration of the shape and average size of proto-imogolite for different synthesis leading toward allophane and imogolite. This scenario with incompatible initial nanostructures could legitimate the use of "proto-imogolite" for ILS nanostructures above the sphere/tube size threshold and "proto-imogolite allophane" for ILS nanostructures below the sphere/tube size threshold.

\section{Conclusions}

A mechanical model based on flexible octahedra and tetrahedra associated in the ILS enables to mimic the mechanical behavior of imogolite. Indeed, the model reproduces the radius of minimum energy, the 
rigidity and the repeat distance along the c-axis of hybrid imogolite with bond parameters having reasonable values. In order for such model to reproduce the characteristic of normal Si-OH imogolite, selection of unrealistic bond parameters would have to be performed. This further demonstrates the fact that $\mathrm{H}$ bond networks and electrostatic interactions are very important to explain the strong curvature of pristine imogolite. Using this model for the simulation of single objects without the use of boundary conditions, a size threshold for which the structure of minimum energy goes from a spherical to a tubular shape is observed. For the chosen parameters, this size threshold is found at about $4 \mathrm{~nm}$. This value is slightly smaller than the size obtained experimentally for proto-imogolite in the synthesis of imogolite nanotubes. Finally, we make the hypothesis that the average size of the proto-imogolite initially formed at the expense of an amorphous phase controls the shape of the final objects (imogolite or allophane).

\section{Acknowledgements}

We would like to thank Clément Levard, Barbara Bonelli, Peng Yuan, Dun-Yen Kang, Nicolas ArancibiaMiranda for all discussions about imogolites which we always appreciate. Special thanks to Rémy Brossard and Florent Malloggi for interesting discussions on rolling of polymer bilayers leading to mutual inspiration and to David Carrière whose ongoing work on up-to-date nucleation theory may well inspire this research area soon. This research has been possible thanks to the PRODIGE grant of the Labex NanoSaclay and PALM (reference: ANR-10-LABX-0035) and also with the funding of the DIM Nano-K of the Region Paris-lles de France for the HIPPOP project.

\section{Notes and references}

Abidin, Z., Matsue, N., Henmi, T., 2007. Differential formation of allophane and imogolite: Experimental and molecular orbital study. J. Comput. Mater. Des. 14, 5-18. doi:10.1007/s10820-006-9022-0

Alvarez-Ramirez, F., 2007. Ab initio simulation of the structural and electronic properties of aluminosilicate and aluminogermanate natotubes with imogolite-like structure. Phys. Rev. B Condens. Matter Mater. Phys. 76, 1-14. doi:10.1103/PhysRevB.76.125421

Amara, M.S., Paineau, E., Rouzière, S., Guiose, B., Krapf, M.-E.M., Taché, O., Launois, P., Thill, A., 2015. Hybrid, Tunable-Diameter, Metal Oxide Nanotubes for Trapping of Organic Molecules. Chem. Mater. 27, 1488-1494. doi:10.1021/cm503428q

Bac, B.H., Song, Y., Kim, M.H., Lee, Y.B., Kang, I.M., 2009. Single-walled hollow nanospheres assembled from the aluminogermanate precursors. Chem. Commun. (Cambridge, United Kingdom) 57405742. doi:10.1039/b908997g

Belloni, L., Thill, A., 2016. Why a 1:1 2D structure tends to roll? A thermodynamic perspective., in: Yuan, P., Thill, A., Bergaya, F. (Eds.), Nanosized Tubular Clay Minerals: Halloysite and Imogolite. Elsevier, p. 778.

Bottero, I., Bonelli, B., Ashbrook, S.E., Wright, P. a, Zhou, W., Tagliabue, M., Armandi, M., Garrone, E., 2011. Synthesis and characterization of hybrid organic/inorganic nanotubes of the imogolite type and their behaviour towards methane adsorption. Phys. Chem. Chem. Phys. 13, 744-750. doi:10.1039/c0cp00438c

Cradwick, P.D.G., Farmer, V.C., Russell, J.D., Masson, C.R., Wada, K., Yoshinaga, N., 1972. Imogolite, a 
hydrated aluminium silicate of tubular structure. Nat. Phys. Sci. 240, 187-189.

Creton, B., Bougeard, D., Smirnov, K.S., Guilment, J., Poncelet, O., 2008. Molecular dynamics study of hydrated imogolite. 1. vibrational dynamics of the nanotube. J. Phys. Chem. C 112, 10013-10020. doi:10.1021/jp800802u

Cygan, R.T., Liang, J.-J., Kalinichev, A.G., 2004. Molecular Models of Hydroxide, Oxyhydroxide, and Clay Phases and the Development of a General Force Field. J. Phys. Chem. B 108, 1255-1266. doi:10.1021/jp0363287

Demichelis, R., Noël, Y., D'Arco, P., Maschio, L., Orlando, R., Dovesi, R., 2010. Structure and energetics of imogolite: a quantum mechanical ab initio study with B3LYP hybrid functional. J. Mater. Chem. 20, 10417. doi:10.1039/c0jm00771d

Denaix, L., Lamy, I., Yves, J., 1999. Synthetic Colloidal Amorphous Aluminosilicates and Their Precursors $158,315-325$.

Exley, C., Schneider, C., Doucet, F.J., 2002. The reaction of aluminium with silicic acid in acidic solution: An important mechanism in controlling the biological availability of aluminium? Coord. Chem. Rev. 228, 127-135. doi:10.1016/S0010-8545(02)00077-2

Farmer, V.C., Fraser, A.R., Tait, J.M., 1977. Synthesis of imogolite: a tubular aluminium silicate polymer. J. Chem. Soc. Chem. Commun. 13, 462,463.

Georgakilas, V., Perman, J.A., Tucek, J., Zboril, R., 2015. Broad Family of Carbon Nanoallotropes: Classification, Chemistry, and Applications of Fullerenes, Carbon Dots, Nanotubes, Graphene, Nanodiamonds, and Combined Superstructures. Chem. Rev. 115, 4744-4822. doi:10.1021/cr500304f

González, R.I., Ramírez, R., Rogan, J., Valdivia, J.A., Munoz, F., Valencia, F., Ramírez, M., Kiwi, M., 2014. Model for Self-Rolling of an Aluminosilicate Sheet into a Single-Walled Imogolite Nanotube. J. Phys. Chem. C 118, 28227-28233. doi:10.1021/jp508637q

Guimarães, L., Enyashin, A.N., Frenzel, J., Heine, T., Duarte, H.A., Seifert, G., 2007. Imogolite nanotubes: Stability, electronic, and mechanical properties. ACS Nano 1, 362-368. doi:10.1021/nn700184k

Henmi, T., Wada, K., 1976. Morphology and composition of allophane. Am. Mineral. 61, 379-390.

Koenderink, G.H., Kluijtmans, S.G.J.., Philipse, A.P., 1999. On the Synthesis of Colloidal Imogolite Fibers. J. Colloid Interface Sci. doi:10.1006/jcis.1999.6323

Konduri, S., Mukherjee, S., Nair, S., 2006. Strain energy minimum and vibrational properties of singlewalled aluminosilicate nanotubes. Phys. Rev. B - Condens. Matter Mater. Phys. 74, 1-4. doi:10.1103/PhysRevB.74.033401

Lee, S.U., Choi, Y.C., Youm, S.G., Sohn, D., 2011. Origin of the strain energy minimum in imogolite nanotubes. J. Phys. Chem. C 115, 5226-5231. doi:10.1021/jp108629z

Levard, C., Rose, J., Thill, A., Masion, A., Doelsch, E., Maillet, P., Spalla, O., Olivi, L., Cognigni, A., Ziarelli, F., Bottero, J.Y., 2010. Formation and growth mechanisms of imogolite-like aluminogermanate nanotubes. Chem. Mater. 22, 2466-2473. doi:10.1021/cm902883p 
Li, L., Xia, Y., Zhao, M., Song, C., Li, J., Liu, X., 2008. The electronic structure of a single-walled aluminosilicate nanotube. Nanotechnology 19, 175702. doi:10.1088/0957-4484/19/17/175702

Maillet, P., Levard, C., Spalla, O., Masion, A., Rose, J., Thill, A., 2011. Growth kinetic of single and doublewalled aluminogermanate imogolite-like nanotubes: an experimental and modeling approach. Phys. Chem. Chem. Phys. 13, 2682-2689. doi:10.1039/c0cp01851a

Mukherjee, S., Bartlow, V.M., Nair, S., 2005. Phenomenology of the growth of single-walled aluminosilicate and aluminogermanate nanotubes of precise dimensions. Chem. Mater. 17, 49004909. doi:10.1021/cm0505852

Plimpton, S., 1995. Fast Parallel Algorithms for Short-Range Molecular Dynamics. J. Comput. Phys. 117, 1-19. doi:10.1006/jcph.1995.1039

Russell, J.D., 1969. Imogolite: A Unique Aluminosilicate. Clay Miner. 8, 87-99. doi:10.1180/claymin.1969.008.1.09

Taché, O., Rouzière, S., Joly, P., Amara, M., Fleury, B., Thill, A., Launois, P., Spalla, O., Abécassis, B., 2016. MOMAC: a SAXS/WAXS laboratory instrument dedicated to nanomaterials. J. Appl. Crystallogr. 49, 1624-1631. doi:10.1107/S1600576716012127

Tamura, K., Kawamura, K., 2002. Molecular dynamics modeling of tubular aluminum silicate: Imogolite. J. Phys. Chem. B 106, 271-278. doi:10.1021/jp0124793

Thill, A., Guiose, B., Bacia-Verloop, M., Geertsen, V., Belloni, L., 2012. How the Diameter and Structure of (OH) 3 Al 203 Si x Ge 1-x OH Imogolite Nanotubes Are Controlled by an Adhesion versus Curvature Competition. J. Phys. Chem. C 116, 26841-26849. doi:10.1021/jp310547k

Wada, S. ichiro, 1987. IMOGOLITE SYNTHESIS AT 25 degree C. Clays Clay Miner. 35, 379-384. doi:10.1346/CCMN.1987.0350508

Wada, S., Wada, K.O.J., 1982. Effects of substitution of germanium for silicon in imogolite . Clays Clay Miner. 30, 123-128.

Yucelen, G.I., Choudhury, R.P., Leisen, J., Nair, S., Beckham, H.W., 2012. Defect Structures in Aluminosilicate Single-Walled Nanotubes: A Solid-State Nuclear Magnetic Resonance Investigation. J. Phys. Chem. C 116, 17149-17157. doi:10.1021/jp3059728

Yucelen, G.I., Kang, D.-Y., Schmidt-Krey, I., Beckham, H.W., Nair, S., 2013. A generalized kinetic model for the formation and growth of single-walled metal oxide nanotubes. Chem. Eng. Sci. 90, 200-212. doi:10.1016/j.ces.2012.12.025

Zhao, M., Xia, Y., Mei, L., 2009. Energetic minimum structures of imogolite nanotubes: A first-principles prediction. J. Phys. Chem. C 113, 14834-14837. doi:10.1021/jp9056169 
